\title{
Vom Elfenbeinturm auf die Straße und zurück - Kompass für ein solidarisches Quartier
}

\author{
Maximilian Hellriegel ${ }^{1}$. Sara Schmitt Pacífico ${ }^{1}$ \\ Eingegangen: 28. April 2020 / Überarbeitet: 18. Oktober 2020 / Angenommen: 22. Oktober 2020 / Online publiziert: 24. November 2020 \\ (c) Der/die Autor(en) 2020
}

\section{Zusammenfassung}

Vor dem Hintergrund der zunehmenden Veränderung des städtischen Lebensumfeldes durch Gentrifizierung, investorenfreundliche Stadtpolitik, Privatisierung öffentlicher Räume, Einsparung öffentlicher Investitionen und den Abbau demokratischer Beteiligungsinstrumente haben wir uns gefragt: Wie könnte eine solidarische Stadt der Zukunft aussehen? Welche Gegenentwürfe zu aktuell herrschenden Paradigmen in der Stadtentwicklung zeigen uns Wege aus der Alternativlosigkeit hin zu einer solidarischen Praxis auf Quartiersebene? Im Rahmen einer angewandten kritischen Geografie möchten wir zeigen, dass es eine Vielzahl an Projekten und Initiativen gibt, die die Kreativlosigkeit, zu der uns der Neoliberalismus erzogen hat, durchbrechen und an konkreten Ideen und deren praktischer Umsetzung arbeiten. Als theoretische Annäherung dafür setzen wir uns mit Utopien und deren Potenzialen für eine politische Praxis auseinander. Da wir selbst im Kontext stadtpolitischer Gruppen engagiert sind, nutzen wir die aktivistische Stadtforschung als methodischen Rahmen unserer Forschung. Daraus entstanden ist ein Faltblatt, der „Kompass für ein solidarisches Quartier“, welcher als aktivistisches Werkzeug und Ideengeber für die konkrete Umsetzung transformativer Stadtpolitik dienen soll.

\section{From the ivory tower onto the street and back-Compass for a solidary neighborhood}

\begin{abstract}
Against the background of the increasing changes in the urban environment due to gentrification, investor-friendly urban policies, privatization of public spaces, urban austerity policies and the dismantling of democratic participation instruments, we asked ourselves: what could a future solidary city look like? Which counterprojects to currently prevailing paradigms in urban development lead us out of a lack of alternatives towards a practice of solidarity at the neighborhood level? Within the framework of an applied critical geography, we would like to show that there are a multitude of projects and initiatives that work on concrete ideas and their practical implementation and break through the lack of creativity that neoliberalism has raised us to. As a theoretical approach to this we deal with the concept of utopias and their potentials for political practice. Since we ourselves are involved as political activists on an urban level, we use activist urban research as the methodological framework for our study. Our results are collected in a leaflet the "Compass for a solidary neighborhood", which is intended to serve as an activist tool and source of ideas for the concrete implementation of transformative urban policy.
\end{abstract}

Maximilian Hellriegel

solidarischerkompass@systemli.org

$\triangle$ Sara Schmitt Pacífico

solidarischerkompass@systemli.org

1 Goethe-Universität Frankfurt am Main, Frankfurt am Main, Deutschland

\section{Kompass für ein solidarisches Quartier - wie aus Theorie Praxis werden kann}

Raus aus dem Elfenbeinturm - rein in die Stadt! Der Neoliberalismus mit dem Mantra der Privatisierung und Individualisierung erschien viele Jahre als alternativloses Credo in städtischem Regierungshandeln (Swyngedouw 2013), was sich vielerorts in Stadtentwicklungsprojekten und Aufwertungsmaßnahmen niederschlägt, die sich vornehmlich privaten Kapitalinteressen unterordnen (Harvey 
2013). Durch die sich zuspitzenden Konflikte um öffentliche Räume und Wohnraum in den urbanen Ballungsräumen erwuchs in der vergangenen Dekade eine stetig wachsende Bewegung stadtpolitischer Initiativen (Vogelpohl et al. 2017), die eine Reihe von Gegenentwürfen zum gesellschaftlichen Status quo mobilisieren konnten. Diese Strukturen einer solidarischen Stadtentwicklung stehen im Fokus des vorliegenden Beitrags, der auf den Ergebnissen einer gemeinsamen Masterarbeit der Autorin und des Autors fußt. Dabei steht die Frage im Vordergrund, welche Gegenentwürfe zu aktuell herrschenden Paradigmen in der Stadtentwicklung uns Wege hin zu einer solidarischen Praxis auf Quartiersebene zeigen. Dieser Frage möchten wir mit den Mitteln einer angewandten kritischen Geografie und in Form einer aktivistischen Forschung nachgehen. Im Fokus unserer Untersuchung stehen Ansätze, die sich den Tendenzen einer neoliberalen Stadtentwicklung mit konkreten Strategien und Instrumenten entgegensetzen und solidarische Wege beschreiten. Ziel ist es, stadtpolitische Transformationsprozesse wissenschaftlich zu verhandeln, dabei aktivistische Initiativen zu begleiten und gesellschaftliche Veränderungen in der Sphäre der kritischen Wissenschaft voranzutreiben. Wir nehmen diesen Beitrag zum Anlass, ,neue Formen gesellschaftlicher Ermächtigung in den Nischen, Freiräumen und an den Rändern der kapitalistischen Gesellschaft" aufzuspüren (Wright 2017, S. 415) und diese sichtbar zu machen.

Den theoretischen Zugang unserer Forschung liefert eine Auseinandersetzung mit dem Begriff der Utopie sowie dessen Bedeutung für angewandte Forschung. Methodisch versuchen, wir klassische Pfade akademischer Wissensproduktion zu verlassen, und widmen uns den Ansätzen aktivistischer Stadtforschung. Die Ergebnisse der Arbeit haben wir grafisch so aufgearbeitet, dass sie auch außeruniversitär nutzbar sind und gesellschaftliche Debatten um eine Stadt für Alle anstoßen. So ist der „Kompass für ein solidarisches Quartier“ in Form eines Faltblatts entstanden, der als konkretes Praxisbeispiel einer angewandten kritischen Geografie dient.

\section{Kritik und dann? Utopien!}

Der Fokus kritischer Wissenschaft liegt auf der Analyse gesellschaftlicher Kräfte- und Herrschaftsverhältnisse - positive Zukunftsbilder einer besseren Gesellschaft finden dort nur selten Platz. Mit unserem Beitrag möchten wir den Versuch starten, uns auf Zukunftsbilder zu konzentrieren und diese auf einen herrschaftskritischen Hintergrund zu zeichnen. Dabei hilft uns ein Blick auf die Denkfigur der Utopie.

Der von Ernst Bloch geprägte Begriff der konkreten Utopien (1985a) verweist auf die Bedeutung von Utopien für eine politische Praxis und auf damit entstehende Möglich- keitsräume für gesellschaftliche Transformationsprozesse. Diesen Ausführungen folgend, begreifen wir „die wesentliche Funktion, die dann Utopie hat, [...] [als] eine Kritik am Vorhandenen“, mit einem gleichzeitigen Augenmerk auf darin verborgene Möglichkeiten und Hoffnungen (Bloch 1985b, S. 362). Es geht uns dabei um eine „Negation des Bestehenden, Intention auf Besseres, Konkretion von Möglichkeiten [und] Motivation von Aktivitäten“ (Neupert-Doppler 2015, S. 179-180). David Harvey (2000) knüpft an diese Arbeiten an, indem er in seinem Werk „Spaces of Hope“ den Ansatz der konkreten Utopien für Debatten in der kritischen Geografie aufbereitet. Er zeigt auf, dass utopisches Denken und Bewusstsein in konkreten räumlichen Möglichkeiten verankert sein kann - in sog. „spaces of hope“. Jene Orte haben die Fähigkeit, durch die in ihnen verankerten räumlichen Utopien den kapitalistischen Diskurs umzuschreiben (Harvey 2000, S. 17). Eric Olin Wright bezeichnet solche Räume als reale Utopien (2017). Dahinter steht der Gedanke, den gesellschaftlichen Status quo dadurch zu verändern, „dass in den Räumen und Rissen innerhalb kapitalistischer Wirtschaften emanzipatorische Alternativen aufgebaut werden und zugleich um die Verteidigung und Ausweitung dieser Räume“ gekämpft wird (Wright 2017, S. 11).

Die „Einheit von Hoffnung und Prozesskenntnis“ (Bloch 1985a, S. 727) - also die kritische Analyse des gesellschaftlichen Status quo bei gleichzeitiger Reflexion sich daraus ergebender Möglichkeitsräume - sehen wir als produktiven Ausgangspunkt für eine angewandte kritische Geografie.

\section{Raus aus dem Elfenbeinturm}

Wir vertreten den Standpunkt, dass eine angewandte kritische Geografie nicht nur klar Position zu gesellschaftlich bedeutsamen Themen beziehen sollte, sondern sich auch an Fragestellungen orientieren sollte, die für soziale Bewegungen eine hohe Relevanz haben. Akademische Wissensproduktion sollte für politische Initiativen nutzbar und zugänglich gemacht werden - ein Ansatz, der sich auch in der Entstehung dieses Beitrags und in unserer eigenen Position widerspiegelt. Wir haben diesen Beitrag als Stadtforscherin und Stadtforscher und als Aktivistin und Aktivist verfasst. (Stadt-)politisch aktiv waren wir zur Zeit der Entstehung der Arbeit in der Gruppe Eine Stadt für Alle! Wem gehört die $A B G$ ? in Frankfurt am Main, die wiederum Teil des bundesweiten Recht auf Stadt-Netzwerkes ist. Ziel war es, eine produktive Verbindung von politischer und wissenschaftlicher Arbeit zu schaffen, ohne dabei den kritischen Blick auf das eigene Handeln zu verlieren. 


\section{Aktivistisch forschen hat Methode}

Anstatt Bewegungen und deren Funktionsweise wissenschaftlich zu erklären, versuchen wir mit unserem Praxisbeispiel einen Beitrag zu gesellschaftlichen Transformationsprozessen zu leisten (Halder 2017, S. 45). Dafür nutzen wir den Ansatz der aktivistischen Stadtforschung (Füllner 2016), welcher die Möglichkeit bietet, sich der „gesamten Bandbreite der Methoden der empirischen Sozialforschung" bedienen zu können (Schipper 2018, S. 15). Die Ergebnisse unserer Forschung sollen nach Veröffentlichung im „Dienst sozialer Bewegungen und politischer Aktivist*innen" stehen (Füllner 2016, S. 50). Nichtakademische Wissensproduktion von sozialen Bewegungen in Blogs, Broschüren, Flyern etc. erkennen wir im Sinne eines „wechselseitigen Lernprozesses“ als Expertise an (Schipper 2018, S. 15). Die von uns untersuchten Initiativen und deren Arbeitsfelder haben wir als reale Utopien definiert; ihr Wissen in gedruckter oder digitaler Form lieferte die Grundlage unseres Beitrags. Zusätzlich haben wir uns mit einzelnen Initiativen persönlich getroffen, um der Frage nachzugehen, wie ähnliche Projekte an anderen Orten vervielfältigt werden können. Gemeinsam haben wir versucht, problemzentriert an dem Kompass zu arbeiten, entscheidend war dafür die Offenlegung unserer Doppelrolle als Wissenschaftlerin und Wissenschaftler und Aktivistin und Aktivist.

Die unterschiedlichen Analysemethoden verfolgen alle das Ziel, gemeinsam mit Aktiven aus stadtpolitischen Gruppen und Initiativen ,etwas zu verändern“ (Halder 2017, S. 38), sie mit unseren Erkenntnissen zu stärken (Füllner 2016, S. 91) und ihr Wissen in die akademische Wissensproduktion aufzunehmen.

In unserem Praxisbeispiel stellen wir reale Utopien, die uns Wege zum „Bau einer neuen Gesellschaft im Gehäuse der alten“ zeigen (Wright 2017, S. 442), in den Vordergrund. Die von uns herangezogenen Initiativen und Basisgruppen vertreten ,radikaldemokratisch-egalitäre und emanzipatorische Ideale“ und vermitteln "gangbare Alternativen zu bestehenden Arrangements" (Wright 2017, S. 222). Dabei haben wir den Fokus auf gegenwärtige Ansätze gelegt, die an einer anderen Art der Stadtentwicklung arbeiten und in unterschiedlichen Feldern für eine Stadt für Alle kämpfen.

\section{Der utopische Raum - das Quartier als Space of Hope}

Als Maßstabsebene für unser Praxisbeispiel haben wir das Quartier gewählt. Diese Perspektive ermöglicht es uns, Fragen, die für eine solidarische Stadtentwicklung von zentraler Bedeutung sind, zu behandeln. Denn ein Quartier kann als Abbild gesamtgesellschaftlicher Entwicklungen interpretiert (Schnur 2008, S. 30) und als räumliche Ebene zwischen Individuum und Gesellschaft gelesen werden (Vogelpohl 2008, S. 72). Um konkrete Ansatzpunkte für ein solidarisches Quartier herauszuarbeiten, haben wir 4 Handlungsfelder definiert, die für gesamtstädtische Prozesse und gesamtgesellschaftliche Entwicklung von Relevanz sind und sich von der Quartiersebene abstrahieren lassen. Die Themen Planen, Wohnen, Freiräume und urbanes Grün und Infrastrukturen bilden die 4 Handlungsfelder, die wir für die Entwicklung des Kompasses heranziehen.

\section{Mit Utopien kritisch denken}

Dem Ansatz von Utopien folgend, haben wir im ersten Arbeitsschritt in den 4 Feldern den Forschungsstand aus herrschaftskritischer Perspektive zusammengefasst. Aus dieser Übersicht werden vielschichtige Handlungsbedarfe für die Frage, wie eine solidarische Stadt aussehen kann, sichtbar. Basierend auf dieser Analyse, haben wir im zweiten Arbeitsschritt stadtpolitische Initiativen ausgewählt, die jene Bedarfe aufgreifen und im Sinne realer Utopien Gegenkonzepte entwickeln. Dabei haben wir uns mit etwa 25 Gruppen und Initiativen aus dem deutschsprachigen Raum auseinandergesetzt, darunter u. a. Stadt von Unten Berlin, die PlanBude Hamburg, das Solidarity Cities-Netzwerk, das Netzwerk Care Revolution oder die Poliklinik in Hamburg.

Was können wir von der Herangehensweise und Praxis dieser Beispiele lernen? Was ist übertragbar? Welche allgemeingültigen Prinzipien für ein solidarisches Quartier lassen sich ableiten? Basierend auf diesen Fragen, haben wir in den 4 Feldern wichtige Teilaspekte, die Wege hin zu einer gesellschaftlichen Emanzipation aufzeigen, herausgearbeitet und im „Kompass für ein solidarisches Quartier“ zusammenfassend dargestellt.

\section{Ein Blick auf die herrschenden Verhältnisse und real- utopische Antworten}

Die kapitalistische Urbanisierung neigt fortwährend dazu „die Stadt als soziales, politisches und lebenswertes Gemeingut zu zerstören“ (Harvey 2013, S. 148). Die katastrophalen Folgen dieser Zerstörung lassen sich in allen 4 beschriebenen Handlungsfeldern ablesen - sei es durch die Unterordnung von (Stadt-)Planungsprozessen unter Kapitalverwertungsinteressen, die sich zuspitzende soziale Krise auf dem Wohnungsmarkt, die Kommerzialisierung öffentlicher Räume, die Zerstörung von Grünräumen oder die Auslagerung öffentlicher Infrastrukturen in den Privatsektor. Die Ergebnisse unseres ersten Analyseschrittes lassen Tendenzen erkennen, die wir im Rahmen des vorliegenden Beitrags nur stichwortartig unter Kommodifizierung, Priva- 
tisierung, Deregulierung und Individualisierung zusammenfassen können.

Die von uns ausgewählten realen Utopien setzen an diesen Aspekten an und stellen den beschriebenen Tendenzen alternative Praktiken, Entwürfe und Modelle entgegen. Die dafür herangezogenen Strategien sind je nach Themenfeld unterschiedlich, verfolgen aber gemeinsame, übergeordnete Ziele und lassen sich in den Begriffen wie Dekommodifizierung, Demokratisierung, Vergesellschaftung und Solidarität bündeln.

Solidarität beschreibt die Beziehung zwischen Menschen, die von kollektivem Handeln und gegenseitigem Helfen geprägt ist. Die alltägliche Praxis der ausgewählten realen Utopien zeigt, wie „solidarische Beziehungsweisen“ zwischen unterschiedlichen Akteuren eingegangen werden können (Adamczak 2017, S. 32). Sie sind von ,utopischer Praxis“ und von ,praktisch-utopischen Forderungen“" geprägt, die ,eine bedürfnisorientierte, [...] solidarische Beziehungsweise gegen die tauschwertorientierte, kapitalistische" setzen (Adamczak 2017, 2018, S. 32-33). Das Knüpfen dieser Beziehungen beschreibt einen kleinteiligen Prozess, dessen einzelne Teile in ihrer Summe den Weg zu einer emanzipatorischen Gesellschaft ebnen können. Dekommodifizierung bezieht sich primär auf Güter, Dienstleistungen und Infrastrukturen, die ,ganz oder teilweise den Marktlogiken“ entzogen und ,aus Verwertungs- bzw. Kapitalkreisläufen" herausgenommen werden (Schipper 2018, S. 1). Darin spielt Demokratisierung, als ,radikalreformistische Transformationsperspektive" (Martin und Wissel 2018, S. 213) eine entscheidende Rolle, da dekommodifizierte Bereiche nicht allein dem Staat untergeordnet, sondern in Verwaltungs- und Organisationsformen übertragen werden sollen, in denen die Nutzerinnen und Nutzer über öffentliche Güter - von Gesundheit über Energie bis hin zu Infrastrukturen wie Wohnen und Mobilität - mitbestimmen können. Zivilgesellschaftliche Kräfte sollen diese Güter politisch steuern können. Betrachtet man das Zusammenwirken von Dekommodifizierung und Demokratisierung, resultiert dies in Prozessen der Vergesellschaftung. Damit werden zugleich Ziel und Prozess einer kollektiven Wiederaneignung von Gütern und Infrastrukturen, die sich durch die kapitalistische Wirtschaftsweise in Privatbesitz befinden und unter Verwertungszwang stehen, beschrieben.

Die 4 genannten Aspekte sind zentrale Bedingungen für die Verwirklichung realer Utopien und entscheidende Schritte in Richtung gesellschaftlicher Transformation. Die real-utopischen Beispiele füllen diese abstrakten Begriffe mit Leben. Ihre Praktiken zeigen uns, wie wir gemeinsam an einer Stadt für Alle arbeiten können und wie diese von allen Bewohnerinnen und Bewohnern gestaltet, produziert und genutzt werden kann.

\section{Poliklinik Veddel - ein real-utopisches Beispiel}

Anhand der Poliklinik Veddel in Hamburg möchten wir beispielhaft einzelne Antworten auf die Frage, wie städtische und soziale Infrastrukturen neu gedacht und demokratisch organisiert werden können, veranschaulichen. Die Poliklinik Veddel ist ein Gesundheits- und Stadtteilzentrum in Veddel. Die Hamburger Gruppe nutzt die seit 2004 bestehende Möglichkeit, ein sog. medizinisches Versorgungszentrum zu gründen, um lokal ein „Gesundheitssystem von unten“ aufzubauen und einen „Ort des Treffpunkts und Austausches, der Bildung und kulturellen Aktion für Menschen aus der Nachbarschaft" zu schaffen (Poliklinik Veddel 2015, S. 14-15). Basierend auf der Analyse, dass ,politische und soziale Faktoren wie Mietsteigerungen, geringes Einkommen, prekäre Beschäftigungsverhältnisse, Rassismus oder Altersarmut" die Gesundheit beeinflussen, versucht die Initiative, die „Ökonomisierung des ambulanten Sektors aufzuhalten" und stattdessen ein Solidarsystem aufzubauen (Poliklinik Veddel 2018). Dies geschieht durch „kontinuierliche Gemeinwesenarbeit in Form von Präventionsprojekten“, mit denen versucht wird, „Gesundheitsproblemen auch kollektiv zu begegnen" (Poliklinik Veddel 2018). Dabei sollen einerseits Statusunterschiede innerhalb der Gesundheitsberufe abgebaut werden, und anderseits soll die Selbstbestimmung der Patientinnen und Patienten gestärkt werden. Mit einem ,starken Fokus auf gesellschaftlich krankmachende Faktoren wie ungleiche Einkommen, Wohnverhältnisse, Rassismus, Umweltbedingungen und Bildung" werden Gesundheit und Krankheit individuell und gesellschaftlich-kollektiv verhandelbar gemacht (Poliklinik Veddel 2018). Damit ,soll der neoliberalen Erzählung von Eigenverantwortung, individuellen Risikofaktoren, und Verhaltensprävention begegnet werden“ (Poliklinik Veddel 2018).

Die Arbeit der Aktivistinnen und Aktivisten basiert auf einer umfassenden Sozialraumanalyse und dem engen Kontakt zu den Akteurinnen und Akteuren im Stadtteil. Entscheidend dabei sind ein breit angelegtes Verständnis von Gesundheit und die Analyse des Status quo im Gesundheitswesen. Den gilt es nach Meinung der Poliklinik, grundlegend zu verändern

mit einem Konzept, das sich den Herausforderungen unserer Zeit stellt, in dem das Postmigrantische als Stärke und nicht als Problem gesehen wird, in dem die krankmachenden Verhältnisse und nicht nur das individuelle Verhalten ins Blickfeld geraten, in dem gemeinsam über Berufsgrenzen und Rollenbilder hinweg nach kollektiven Lösungsstrategien gesucht wird, in dem eine Kultur der Teilhabe und des Willkommens eine Antwort auf Krankheit ist. (Poliklinik Veddel 2018) 
Primäre Gesundheitsversorgung, Kollektivierung der gesundheitlichen Problemlage im Stadtteil und die Veränderung der Verhältnisse sind Wege, durch die an der Verbesserung der Stadtteilgesundheit gearbeitet wird. Es wird nicht nur klassische medizinische Versorgung angeboten, sondern auch Rechtsberatung, Bildungsarbeit und ein Pflegedienst.

Gemeinsam mit den Patientinnen und Patienten, Stadtteilbewohnerinnen und Stadtteilbewohnern und den Mitarbeitenden der Poliklinik wird an der Repolitisierung des Themas Gesundheit gearbeitet (Poliklinik Veddel 2015, S. 16), dabei sieht die Poliklinik ihre Aufgabe darin, die

Nutzer*innen der Poliklinik und die Bewohner*innen des Stadtteils in ihrem Recht auf gesellschaftliche Teilhabe und Mitgestaltung der Verhältnisse zu unterstützen und gemeinsam reale Utopien für eine Verbesserung der Lebensverhältnisse zu entwerfen, für deren Umsetzung zu kämpfen und sie einfach zu leben. (Poliklinik Veddel 2018)

Auf der Suche nach Grundrissen für ein solidarisches Quartier haben wir Projekte gefunden, die ihre konkrete Stadtteilarbeit mit strukturellen Fragen verbinden und so gesellschaftliche Transformation in Gang setzen. Die Poliklinik Veddel, von uns definiert als reale Utopie, zeigt wie auf lokaler Ebene „,neue Formen der sozialmedizinischen, interdisziplinären Versorgung erprobt" werden können (Rakowitz 2017, S. 4) und gleichzeitig an der „Demokratisierung des Gesundheitssystems“ als Ganzes gearbeitet werden kann (Rakowitz 2017, S. 36-37).

Die Initiative steht an dieser Stelle als ein Exempel für die vielen realen Utopien, die wir im Rahmen unserer Forschung aufgespürt und analysiert haben.

\section{Kompass für ein solidarisches Quartier - das Praxisbeispiel}

Der „Kompass für ein solidarisches Quartier“ stellt das praktische Beispiel der Umsetzung einer angewandten kritischen Geografie dar und gibt einen spielerisch-grafischen Überblick über die von uns identifizierten realen Utopien sowie deren politische Ansätze, Konzepte und Erfahrungen (Abb. 1). Im Sinne einer aktivistischen Stadtforschung haben wir die Forschungsergebnisse auf ein DIN-A1-großes Faltblatt so aufgearbeitet, dass diese auch für Menschen außerhalb eines akademischen Kontexts verständlich und nutzbar sein können. Der Kompass als Ganzes bildet Ansätze für ein positives Zukunftsbild einer Stadt für Alle ab und soll Initiativen, Projekte und soziale Bewegungen als
Abb. 1 Ausschnitt aus dem Faltblatt „Kompass für ein solidarisches Quartier" - Downloadlink: https://www.rosalux. de/fileadmin/ls_he/dokumente/ Faltblatt_Kompass_f\%C3 $\%$ BCr_eine_solidarisches_ Quartier.pdf
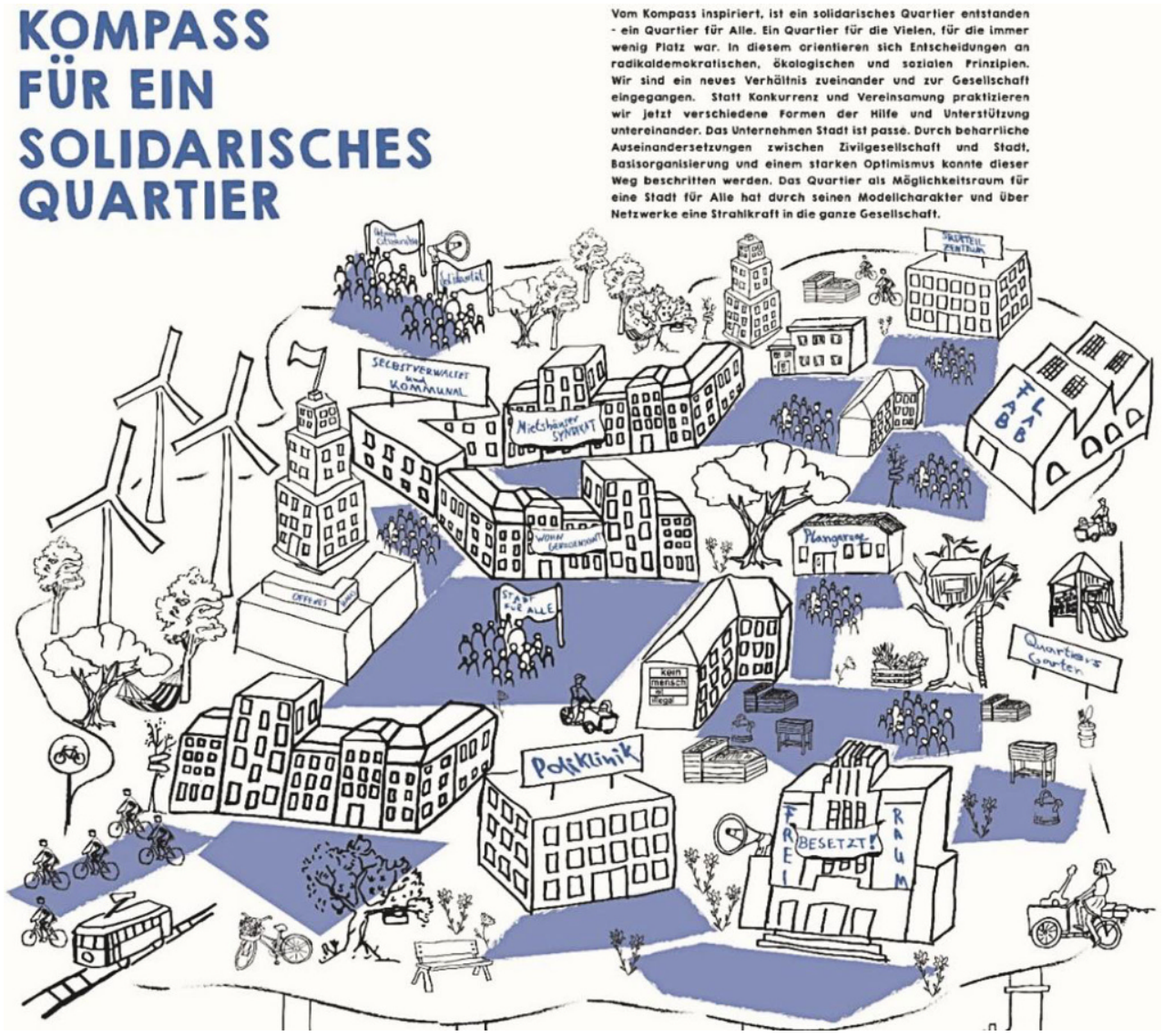
aktivistisches Werkzeug in ihrer Arbeit unterstützen. Die vielen Beispiele regen außerdem zum Nachmachen, Weitermachen und Selbermachen an.

Soll der Ansatz einer angewandten kritischen Geografie ernst genommen werden, braucht es einen langen Atem: Nach der Forschung gilt es, die Ergebnisse für Aktivistinnen und Aktivisten aus der Universität in die Bewegung zu tragen. Notwendig dafür ist u.a. finanzielle Unterstützung. In unserem Fall hat die Rosa Luxemburg Stiftung Hessen den Druck von insgesamt 1000 Exemplaren des Faltblattes finanziert und es uns ermöglicht, unsere Ergebnisse bundesweit auf verschiedenen Foren und Treffen der Recht auf Stadt-Bewegung vorzustellen. Vor allem im Anschluss an die Arbeit ist es unserer Meinung nach von Bedeutung, von Forscherinnen und Forschern wieder zu Aktivistinnen und Aktivisten zu werden und Wissen, Erfahrungen und die uns entgegengebrachte Solidarität wieder in die Bewegung zurück zu tragen.

Der Kompass war Anlass für uns, z. B. in Frankfurt am Main eigene Veranstaltungen zum Thema zu organisieren, uns dort mit stadtpolitischen Gruppen zu vernetzen und gemeinsam über Handlungsoptionen zu diskutieren. Das Faltblatt wird immer dann zu einem Werkzeug, wenn es Debatten anstößt, Handlungsoptionen aufzeigt und zum Mit-/ Weitermachen motiviert.

\section{Die Verhältnisse zum Tanzen bringen}

Ziel unserer Arbeit war es, ein positives Bild für die $\mathrm{Zu}$ kunft unserer Städte nachzuzeichnen, ohne dabei in naivutopische Visionen zu verfallen. Es gilt, herrschende Kräfteverhältnisse zu durchbrechen, den Glauben an Alternativlosigkeit abzulegen und gemeinsam an realen Utopien $\mathrm{zu}$ arbeiten. Wir wollen Wege zu einer Stadt für Alle aufzeigen, hegemoniale Denkblocken aufbrechen, die Verhältnisse zum Tanzen bringen und andere zum Mittanzen einladen (AG links-Netz 2013, S. 74; MEW 1 1976, S. 383). Das von uns skizzierte Faltblatt ist ein Weg für die Geografie, sich in (stadt)politische Diskussionen einzumischen und kritische Forschungsinhalte aus dem Elfenbeinturm der Akademie auf die Straßen und in die Stadtöffentlichkeit zu tragen.

Die in vielen Bereichen mobilisierten Alternativen einer solidarischen Stadtentwicklung von unten zeigen, dass auch in der Praxis fernab universitärer Arbeit ein kritischer Blick auf gesellschaftliche Entwicklungen dabei helfen kann, neue Wege zu gehen. Eine angewandte kritische Geografie ist unserer Meinung nach unverzichtbar, um soziale Innovationen aus Forschung und Gesellschaft in eine konkrete Praxis zu überführen und institutionell anzubinden. Dafür ist es wichtig, die Ressourcen und Freiräume, die Universitäten (noch) bieten, zu nutzen. Die Fusion von Aktivismus und Forschung ist eine Möglichkeit, im Rah- men des akademischen Arbeitens politische Haltung offen einzunehmen und zu kommunizieren und mit wissenschaftlicher Arbeit einen Beitrag zu gesellschaftlicher Transformation zu leisten (Halder 2017, S. 45).

Funding Open Access funding enabled and organized by Projekt DEAL.

Open Access Dieser Artikel wird unter der Creative Commons Namensnennung 4.0 International Lizenz veröffentlicht, welche die Nutzung, Vervielfältigung, Bearbeitung, Verbreitung und Wiedergabe in jeglichem Medium und Format erlaubt, sofern Sie den/die ursprünglichen Autor(en) und die Quelle ordnungsgemäß nennen, einen Link zur Creative Commons Lizenz beifügen und angeben, ob Änderungen vorgenommen wurden.

Die in diesem Artikel enthaltenen Bilder und sonstiges Drittmaterial unterliegen ebenfalls der genannten Creative Commons Lizenz, sofern sich aus der Abbildungslegende nichts anderes ergibt. Sofern das betreffende Material nicht unter der genannten Creative Commons Lizenz steht und die betreffende Handlung nicht nach gesetzlichen Vorschriften erlaubt ist, ist für die oben aufgeführten Weiterverwendungen des Materials die Einwilligung des jeweiligen Rechteinhabers einzuholen.

Weitere Details zur Lizenz entnehmen Sie bitte der Lizenzinformation auf http://creativecommons.org/licenses/by/4.0/deed.de.

\section{Literatur}

Adamczak B (2017) Beziehungsweise Revolution. 1917, 1968 und kommende. Suhrkamp, Berlin

Adamczak B (2018) „Ich halte das anti-utopische Bilderverbot für erledigt“. Gespräch mit Bini Adamczak. In: Neupert-Doppler A (Hrsg) Konkrete Utopien. Schmetterling, Stuttgart, S 23-38

Bloch E (1985a) Das Prinzip Hoffnung. Suhrkamp, Frankfurt am Main

Bloch E (1985b) Tendenz, Latenz, Utopie. Suhrkamp, Frankfurt am Main

Füllner J (2016) Aktivistische Stadtforschung. In: Belina B, Naumann M, Strüver A (Hrsg) Handbuch kritische Stadtgeographie. Westfälisches Dampfboot, Münster, S 86-91

Halder S (2017) Gemeinsam die Hände dreckig machen. Aktionsforschungen im aktivistischen Kontext urbaner Gärten und kollektiver Kartierungen. transcript, Bielefeld

Harvey D (2000) Spaces of hope. Edinburgh Univ. Press, Edinburgh

Harvey D (2013) Rebellische Städte. Vom Recht auf Stadt zur urbanen Revolution. Suhrkamp, Berlin

links-Netz AG (2013) Sozialpolitik als Bereitstellung einer Sozialen Infrastruktur. In: Hirsch J, Brüchert O, Krampe E (Hrsg) Sozialpolitik anders gedacht. Soziale Infrastruktur. VSA, Hamburg, S 50-74

Martin D, Wissel J (2018) Soziale Infrastruktur als sozialpolitisches Transformationskonzept. In: Brand U, Görg C (Hrsg) Zur Aktualität der Staatsform. Die materialistische Staatstheorie von Joachim Hirsch. Nomos, Baden-Baden, S 201-218

MEW 1 (1976) Zur Kritik der Hegelschen Rechtsphilosophie. In: In: Karl Marx / Friedrich Engels - Werke, Band 1, Dietz Verlag, Berlin

Neupert-Doppler A (2015) Utopie. Vom Roman zur Denkfigur. Schmetterling Verlag, Stuttgart

Poliklinik Veddel (2015) Poliklinik - eine Konzeptstreitschrift. Eine kontroverse aus der Vergangenheit. In: Verein demokratischer Ärztinnen und Ärzte (Hrsg) Gesundheit braucht Politik. Soziale Determinanten von Gesundheit, S 14-16

Poliklinik Veddel (2018) Stadtteil-Gesundheits-Zentrum. http://poli klinik1.org/. Zugegriffen: 10. Apr. 2018 
Rakowitz N (2017) Gesundheit ist eine Ware. Mythen und Probleme des kommerzialisierten Gesundheitswesens. luxemburg argumente Nr. 6. Rosa-Luxembur-Stiftung, Berlin

Schipper S (2018) Wohnraum dem Markt entziehen? Wohnungspolitik und städtische soziale Bewegungen in Frankfurt und Tel Aviv. VS, Wiesbaden

Schnur O (Hrsg) (2008) Quartiersforschung. Zwischen Theorie und Praxis. VS, Wiesbaden

Swyngedouw E (2013) Die postpolitische Stadt. Sublurban Z Krit Stadtforsch 1(2):141-158. https://doi.org/10.36900/suburban. v1i2.100

Vogelpohl A (2008) Stadt der Quartiere? Das Place-Konzept und die Idee von urbanen Dörfern. In: Schnur O (Hrsg) Quartiersfor- schung. Zwischen Theorie und Praxis. VS, GWV, Wiesbaden, S 69-87

Vogelpohl A, Vollmer L, Vittu E, Brecht N (2017) Die Repolitisierung des Wohnens. Städtische soziale Bewegungen für ein Recht auf Wohnen und auf Stadt im Hamburg, Berlin, Jena und Leipzig. In: Kadi J, Schipper S, Schönig B (Hrsg) Wohnraum für alle?! Perspektiven auf Planung, Politik und Architektur. transcript, Bielefeld, S 102-131

Wright E (2017) Reale Utopien. Wege aus dem Kapitalismus. Suhrkamp, Berlin 